\title{
Hip Arthroscopy for Femoroacetabular Impingement: Minimal Clinically Important Difference Rates Decline From 1- to 5-Year Outcomes
}

\author{
Berkcan Akpinar, M.D., Lawrence J. Lin, B.A., David A. Bloom, B.S., and \\ Thomas Youm, M.D.
}

\begin{abstract}
Purpose: To correlate patient-reported outcomes (PROs) and minimal clinically important difference (MCID) achievement rates after hip arthroscopy for femoroacetabular impingement syndrome (FAI). Methods: Patients with clinically diagnosed FAI who underwent primary hip arthroscopy from September 2012 to March 2014 with a minimum of 5-year outcomes were identified. Patients undergoing labral debridement, microfracture, bilateral procedures, with evidence of dysplasia, Tönnis grade $>1$, and joint space $<2 \mathrm{~mm}$ were excluded. Analysis of variance was used to compare PROs. Survival rates were determined using Kaplan-Meier analysis. Regression analysis identified associations with modified Harris Hip Scores (mHHS), minimal clinically important difference (MCID) rates, and Nonarthritic Hip Scores (NAHS). Results: A total of 85 of 101 eligible consecutive patients (84\% inclusion) (age: $41.4 \pm 14.0$ years; $69 \%$ female, mean body mass index [BMI] $25.0 \pm 4.2$ ) met inclusion and exclusion criteria. Patients underwent labral repair (100\%) and a combination of cam $(86 \%)$ and pincer resection $(99 \%)$. The 5-year survival-to-revision rate was $77 \%$ whereas 5 -year survival rate to total hip arthroplasty was $94 \%$. The 1 -year $(87.4 \pm 13.6)$ and 5-year (84.5 \pm 13.5$)$ mHHS scores were greater versus preoperative scores $(46.3 \pm 11.3, P<.001)$. There was a decrease in MCID rate between $1-y e a r(n=74$, $87 \%)$ and 5 -year $(\mathrm{n}=61,73 \%, P=.019)$ outcomes. The 1 -year $(87.4 \pm 12.7)$ and 5-year (89.2 \pm 15.8$)$ NAHS scores were greater versus preoperative scores $(49.7 \pm 12.7, P<.001)$. Regression demonstrated associations between BMI (MCID: $P=.033$; NAHS: $P=.010$ ), age (mHHS: $P=.031$ ), and cam resection (mHHS: $P=.010$ ) with 5-year outcomes. Conclusions: There is a decline in MCID at 5-year follow-up after hip arthroscopy for FAI. Lower BMI, younger age, and cam resection are associated with positive outcomes. There is excellent index procedure survivability and excellent total hip arthroplasty prevention rate. Level of Evidence: Level IV.
\end{abstract}

$\mathbf{F}$ emoroacetabular impingement syndrome (FAI) is a hip condition associated with pathologic contact of the femoral head and neck with the acetabulum, often times resulting in associated chondral and labral injuries. ${ }^{1,2}$ In a subset of patients, FAI may generate hip pain and mechanical symptoms leading to activity restriction and disability. Lastly, FAI has been associated

From NYU Langone Orthopedic Hospital, New York, New York, U.S.A. The authors report the following potential conflicts of interest or sources of funding: T.Y. reports consultant for Arthrex. Full ICMJE author disclosure forms are available for this article online, as supplementary material.

Received April 26, 2020; accepted September 23, 2020.

Address correspondence to Berkcan Akpinar, M.D., NYU Langone Orthopedic Hospital, 301 East 17th St., Suite 1402, New York, NY 10003. E-mail: Berkcan.Akpinar@nyulangone.org

(C) 2020 THE AUTHORS. Published by Elsevier Inc. on behalf of the Arthroscopy Association of North America. This is an open access article under the CC BY-NC-ND license (http://creativecommons.org/licenses/by-nc-nd/4.0/). 2666-061X/20690

https://doi.org/10.1016/j.asmr.2020.09.025 with early hip osteoarthritis development, further indicating the need for adequate treatment strategies. ${ }^{2,3}$ As a result, FAI has become one of the most common indications for hip arthroscopy. ${ }^{2}$

Hip arthroscopy has demonstrated versatility in the treatment of FAI including cam/pincer/anterior inferior iliac spine subspine pathology, labral tears, chondromalacia, ligamentum teres pathology, adhesive changes, and even early-grade osteoarthritis. ${ }^{3-6}$ Given the variability of pathology associated with FAI, a variety of prognostic preoperative clinical, radiographic, and intraoperative factors have been identified in midterm (5-year) studies including Tönnis grade, joint space narrowing, age, body mass index (BMI), microfracture, and labral tear management. ${ }^{2,6-12}$

Menge et al. $^{2}$ show a $34 \%$ conversion to total hip arthroplasty (THA) rate at 10-year follow-up, albeit in a significantly older population (mean age older than 53 years), with substantial degree of patients with arthritis $(26 \%$ with joint space $<2 \mathrm{~mm})$, and with 
subjects undergoing labral debridement (49\%). ${ }^{2}$ Current mid-term data suggest an index surgery failure rate (of revision arthroscopy or conversion to THA) ranging from $0 \%$ to $86 \%$, suggesting that indications for surgery need further optimization. ${ }^{12-15}$ Also, this range of failure rates suggests there is significant variability in the published cohorts to date..$^{2,6-12}$ Furthermore, only 2 mid-term studies have evaluated minimal clinically important difference (MCID) and patient acceptable symptomatic state (PASS) rates, albeit in an elderly patient cohort older than age 50 years and a strictly microfracture cohort, respectively. ${ }^{10,11}$ Although short-term (6 months to 3.5 years) studies demonstrate additional prognostic variables such as preoperative symptom duration, capsular management in addition to the aforementioned factors, lack of significant follow-up limits clinical relevance to an extent. ${ }^{1,3,6,16-18}$ Lastly, while the majority of studies demonstrate the detrimental effects of labral debridement and microfracture, few studies have published outcomes after excluding these 2 procedures from studied cohorts. ${ }^{2,3,8-10,12}$

The purpose of this study was to correlate patientreported outcomes (PROs) and MCID achievement rates after hip arthroscopy for FAI syndrome. We hypothesized MCID rates would decline despite sustained, improvement of 5-year PROs.

\section{Methods}

\section{Patient Population}

A clinical registry from February 2013 to March 2014 containing patients who had undergone hip arthroscopy for FAI performed by the senior author, a board-certified orthopaedic surgeon with a sports medicine fellowship (T.Y.), was used for this investigation. All patients provided informed consent for participation in this institutional review boardapproved study (institutional review board number i15-00058). A combination of clinical pain in the hip corresponding with provocative physical examination maneuvers (anterior impingement test, Patrick test) alongside radiographic findings allowed initial diagnosis of FAI. Anteroposterior, $45^{\circ}$, and $90^{\circ}$ Dunn-view radiographs were obtained in patients clinically diagnosed with FAI to assess bony pathology whereas chondral or labral pathology was evaluated with advanced imaging: magnetic resonance imaging or magnetic resonance arthrography.

Radiographic inclusion criteria for FAI included a lateral center edge angle (LCEA) $\geq 40^{\circ}$, alpha $(\alpha)$ angle $\geq 60^{\circ}$, proximal acetabular retroversion (i.e., cross-over sign) and focal chondrolabral delamination on magnetic resonance imaging or magnetic resonance arthrography. Exclusion criteria were bilateral hip arthroscopy, a Tönnis grade greater than 1 indicating hip osteoarthritis, joint space $<2 \mathrm{~mm}$ on radiographs, an LCEA $<20^{\circ}$ indicative of hip dysplasia, pediatric patients $(<15$ years old), history of rheumatologic disease, slipped capital femoral epiphysis history, previous hip procedures, intraoperative labral debridement or microfracture, and with follow-up less than 5 years.

Patient demographic and surgical factors were obtained from chart review. Operative notes were reviewed to determine intraoperative labral repair, chondroplasty, or femoral or anterior inferior iliac spine osteochondroplasty. Postoperative radiographs were reviewed to assess for LCEA and $\alpha$ angle.

\section{Surgical Technique and Rehabilitation}

All hip arthroscopy procedures were performed with the patient under general anesthesia. Anterior and anterolateral portal placement was used. Initial diagnostic arthroscopy was performed in all cases to assess pathology after interportal capsulotomy. All tears amenable to repair involving the chondrolabral junction were repaired with suture anchors. Shaving chondroplasty was performed for moderate chondral lesions (Outerbridge grade I-III). Dynamic fluoroscopyassisted femoral or periacetabular osteochondroplasty was performed for cam and pincer lesions, respectively. After removal of any loose bodies, a standard capsule repair was performed followed by portal closures.

All patients received postoperative hip bracing limiting hyperextension and external rotation for 1 week. Weight-bearing was restricted to flat-foot with 2 crutches for the first 4 weeks. Standard postoperative antibiotic (cefalexin $500 \mathrm{mg}$ twice daily for 3 days) and deep-vein thrombosis chemoprophylaxis (aspirin $81 \mathrm{mg}$ twice daily for 2 weeks) was administered and Celebrex (200 mg daily) was administered for heterotopic ossification prevention (6 weeks).

\section{PROs and Follow-Up}

PROs in the form of Nonarthritic Hip Scores (NAHS) ${ }^{19}$ and modified Harris Hip Scores (mHHS $)^{20}$ were determined prospectively starting preoperatively and at 1 year and 5 years postoperatively. Additional mHHS values were obtained with retrospective survey distribution if patients were available and follow-up surgeries were determined using the electronic medical record along with patient interviews. MCID achievement rate was calculated for each follow-up time point using 8-point increase in mHHS as a cutoff for each subject. PASS achievement rate was calculated for each follow-up time point using an absolute mHHS threshold of 74 points for each subject. These MCID and PASS parameters were set as determined in previous studies. ${ }^{21,22}$ If patients underwent repeat procedures, MCID and PASS were recorded as not achieved from time point of revision procedure and 


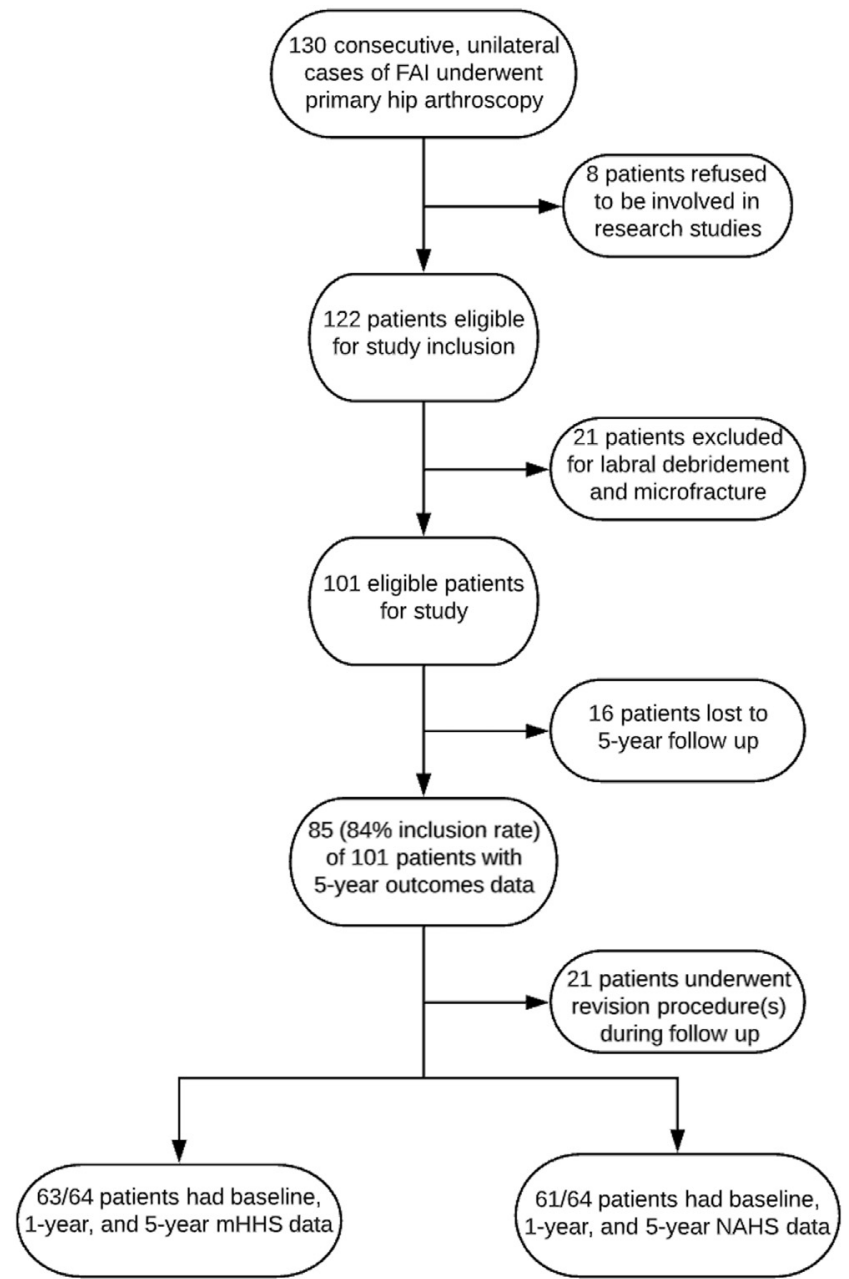

Fig 1. A flowchart depicting inclusion and exclusion of patients for this study. After exclusion of 8 patients refusing research involvement and 21 patients who underwent either labral debridement or microfracture, 101 patients were eligible for the study. The inclusion rate of patients with a minimum of 5 -year follow up for the study was $84 \%$ (81 of 101 patients). Of these 85,21 patients underwent at least 1 revision procedure before 5 -year PRO scores. Of the available 64 patients for PRO comparison between baseline, 1-year, and 5 -year outcomes, 63 had all mHHS datapoints and 61 had all available Nonarthritic Hip Score datapoints. (FAI, femoroacetabular impingement syndrome; mHHS, modified Harris Hip Score; PRO, patient-reported outcomes.)

forward. Surgery failure was defined as revision hip arthroscopy or conversion to a THA.

\section{Statistical Analysis}

Survival Kaplan-Meier analysis (5-year percentage conversion rate; predicted mean survival time, 95\% confidence interval) was performed assessing conversion to any revision surgery as well as time to THA. Descriptive results (mean, standard deviation, range, $\mathrm{n}$ ) of PROs at baseline, 1 year, and 5 years postoperatively were calculated as available. Further, PROs were analyzed with a repeated-measures analysis of variance
(ANOVA) test to assess statistical significance over time. Mauchly's test of sphericity was used to determine homogeneity of data and significance was reported using the Greenhouse-Geisser degrees of freedom correction in cases of violation during repeatedmeasures ANOVA testing. Bonferroni adjustments were used for multiple comparisons. Paired Student $t$ tests were performed to compare preoperative with postoperative $\alpha$ angles and LCEA. $\chi^{2}$ or Fisher exact tests were used to compare PASS and MCID rates at different time points. A multifactorial linear regression analysis was performed assessing 5-year PROs (mHHS or NAHS) as the dependent variable with independent variables including all demographic and intraoperative variables collected (age, BMI, sex, cam resection, pincer resection, preoperative LCEA, postoperative LCEA, preoperative $\alpha$ angle, postoperative $\alpha$ angle, and labral repair). A multifactorial logistic regression analysis also was performed using the aforementioned variables to determine predictive variables in 5-year MCID achievement rate. Lastly, post-hoc power analysis was performed using the 1-year and 5-year PROs to determine observed power $(\beta)$. Using the effect size and standard deviation between 1-year and 5-year PROs for NAHS and mHHS, required sample sizes were also retrospectively calculated for a desired $\beta$ of 0.8 .

\section{Results}

\section{Overall Clinical Outcomes}

A total of 85 of 101 consecutive patients met the inclusion criteria for this study with a mean follow-up of $72.1 \pm 14.7$ months (Fig 1 and Table 1). Forty-one percent $(n=35)$ of the cohort had Tönnis grade 1 joint characteristics on preoperative radiographs.

Table 1. Demographic and Radiographic Characteristics

\begin{tabular}{|c|c|c|}
\hline & & $P$ Value* \\
\hline$\overline{\text { Age, } y, \text { mean } \pm S D}$ & $41.1 \pm 14.0$ & - \\
\hline $\mathrm{BMI}$, mean $\pm \mathrm{SD}$ & $25.0 \pm 4.2$ & - \\
\hline Tönnis grade ${ }^{\dagger}$ & $35,40 \%$ & - \\
\hline $\operatorname{Sex}(n, \%$ female $)$ & $59,69 \%$ & - \\
\hline Laterality ( $\mathrm{n}, \%$ right) & $55,65 \%$ & - \\
\hline Labral repair & $85,100 \%$ & - \\
\hline Chondroplasty & $81,95 \%$ & - \\
\hline Cam resection ${ }^{\ddagger}$ & $73,86 \%$ & - \\
\hline Pincer resection & $84,99 \%$ & - \\
\hline Lateral center edge angle, ${ }^{\circ} \mathrm{C}$ & & $<.001$ \\
\hline Preoperative, mean \pm SD & $41.2 \pm 6.9$ & - \\
\hline Postoperative, mean \pm SD & $32.0 \pm 5.8$ & - \\
\hline Alpha angle, ${ }^{\circ}$ & & $<.001$ \\
\hline Preoperative, mean \pm SD & $62.4 \pm 9.0$ & - \\
\hline Postoperative, mean \pm SD & $54.4 \pm 5.5$ & - \\
\hline
\end{tabular}


A Survival to Initial Revision Procedure

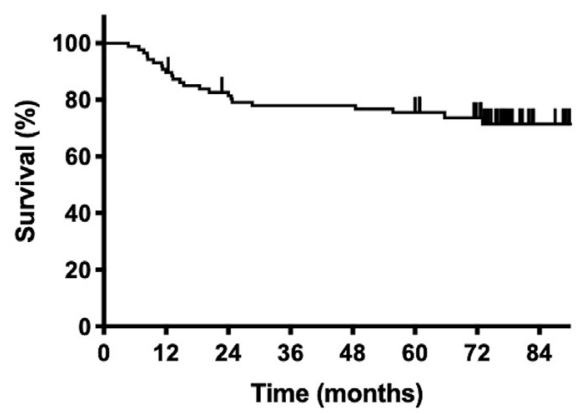

B Survival to Total Hip Arthroplasty

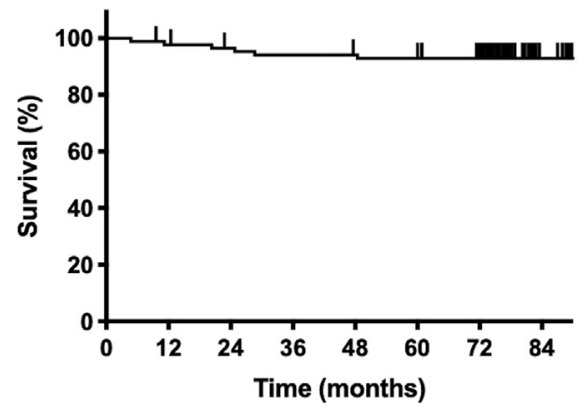

Fig 2. (A) Kaplan-Meier survival analysis demonstrating longevity of index hip arthroscopy before any revision procedure. (B) Kaplan-Meier survival analysis demonstrating longevity of index hip arthroscopy before total hip arthroplasty. Hashmarks indicate subject censoring due to followup loss.
Postoperative LCEA $\left(32.0 \pm 5.8^{\circ}\right)$ and $\alpha$ angle $\left(54.4 \pm 5.5^{\circ}\right)$ improved compared with preoperative LCEA $\left(41.2 \pm 6.9^{\circ}, P<.001\right)$ and $\alpha$ angle $\left(62.4 \pm 9.2^{\circ}\right.$, $P<.001)$ measurements $(80 / 85$ patients with available radiographs) (Table 1$)$. Intraoperatively, a total of 85 labral repairs were performed, 73 cam resections, and 84 pincer resections were performed (Table 1). There was a total of 21 patients in the cohort who needed 1 or more revision procedures within the follow-up period. Revision procedures included 19 repeat hip arthroscopies and 5 conversions to THA (6\% THA rate). There was 1 death during follow-up due to unrelated medical comorbidities. Two patients required more than 1 revision surgery. The 5-year survival rate of the index hip arthroscopy before any revision procedure was 77\% (number at risk: 63) with a mean predicted mean survival time of 74 months $(95 \%$ confidence interval: 67.6-80.7) whereas 5-year survival rate before THA was $94 \%$ (number at risk: 76 ) with a predicted mean survival time of 87 months (95\% confidence interval 84.0-90.8) (Fig 2).

\section{mHHS Outcomes}

Of the patients without subsequent revision surgery, a total of 63 had follow-up allowing for repeated measures comparison at (baseline, 1-year, and 5-year) time points. Baseline mHHS (46.3 \pm 11.3$)$ was significantly lower than 1 -year $(87.4 \pm 13.6, P<.001)$ and 5 -year $(84.5 \pm$ 13.5, $P<.001)$ postoperative outcomes. The mHHS was not different between the 1-year and 5-year time points $(P=.12$, observed $\beta$ : 0.34) (Table 2, Fig 3A). The required sample size for acceptable type II error $(\beta=0.80)$ was 343 (effect size: 2.9 ). A linear regression predicting 5-year mHHS $\left(\mathrm{R}^{2}=0.18, P=.004\right)$ demonstrated age (standardized $\beta$ : $-0.27, P=.031$ ) and cam resection (standardized $\beta$ : $0.33, P=.010$ ) as the only significant predictive variables in the model.

Ultimately, there was no significant differences in the PASS rates achieved between 1-year $(\mathrm{n}=56,66 \%)$ outcomes and 5-year ( $\mathrm{n}=51,61 \%, P=.29)$ outcomes (Fig 4A). However, there was a significant decrease in MCID achievement rate between 1 -year $(\mathrm{n}=74,87 \%)$ and 5 -year $(\mathrm{n}=61,73 \%, P=.019)$ outcomes (Fig $4 \mathrm{~B})$. A binary logistic regression predicting 5-year MCID achievement (Cox and Snell $\mathrm{R}^{2}=0.06$ ) demonstrated BMI (odds ratio $0.87, P=.033$ ) as the only significant predictive variable in the model.

\section{Nonarthritic Hip Score Outcomes}

Of the patients without subsequent revision surgery, a total of 61 had follow-up, allowing for repeated measures comparison at (baseline, l-year and 5-year) postoperative time points. Baseline NAHS (49.7 \pm 12.7 ) was significantly lower than 1-year (87.4 \pm 12.7, $P<.001)$ outcomes and significantly lower than 5-year $(89.2 \pm 15.8, P<.001)$ outcomes. There was no significant difference between 1-year and 5-year NAHS outcomes $(P=.41$, observed $\beta=0.13)$ (Table 1 , Fig $3 \mathrm{~B})$. The required sample size for acceptable type II error $(\beta=0.80$ ) was 984 (effect size $=1.8$ ). A linear regression predicting 5-year NAHS $\left(\mathrm{R}^{2}=0.10\right.$, $P=.010$ ) demonstrated increasing BMI (standardized

Table 2. Descriptive Statistics of Patient-Reported Outcomes

\begin{tabular}{|c|c|c|c|c|c|}
\hline & $\mathrm{N}$ & Minimum & Maximum & Mean & Standard Deviation \\
\hline Baseline mHHS & 63 & 25.2 & 83.3 & 46.3 & 11.3 \\
\hline mHHS 1 year & 63 & 23.8 & 90.0 & 49.7 & 12.7 \\
\hline mHHS 5 years & 63 & 21.3 & 100.0 & 89.2 & 15.8 \\
\hline Baseline NAHS & 61 & 25.2 & 83.3 & 46.3 & 11.3 \\
\hline NAHS 1 year & 61 & 23.8 & 90.0 & 49.7 & 12.7 \\
\hline NAHS 5 years & 61 & 21.3 & 100.0 & 89.2 & 15.8 \\
\hline
\end{tabular}

mHHS, modified Harris Hip Score; NAHS, Nonarthritic Hip Score. 
Fig 3. (A) mHHS across time points (baseline, 1 year, and 5 years). mHHS at baseline was significantly less than mHHS at both 1-year and 5-year scores. Error bars depicting standard deviation. (B) NAHS across time points (baseline, 1 year, and 5 years). NAHS at baseline was significantly less than NAHS at both 1-year and 5-year scores. Error bars depicting standard deviation. ${ }^{*} P<.001$. (mHHS, modified Harris Hip Score; NAHS, Nonarthritic Hip Score.)
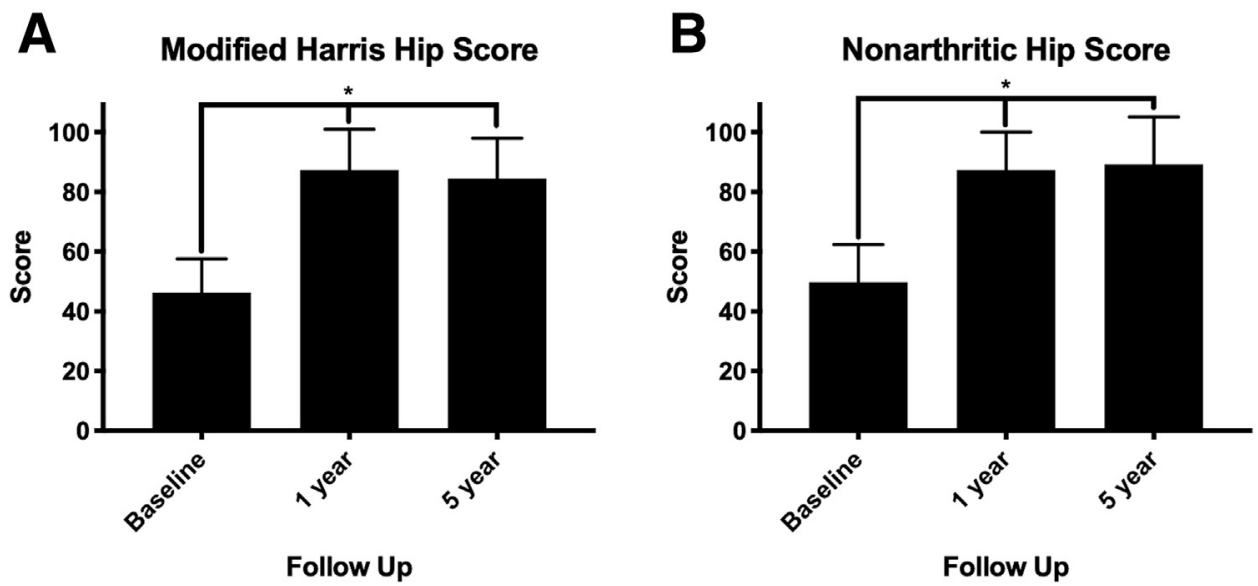

$\beta=-0.33, P=.010$ ) as the only significant predictive variable in the model.

\section{Discussion}

In this study, we have successfully demonstrated that hip arthroscopy for FAI has excellent long-term PROs and longevity outcomes. We report analysis and comparison of sequential follow-up regarding short-term (1-year) and long-term (5-year) outcome characteristics in a high follow-up rate $(82 \%)$ consecutive patient cohort. Notably, there is a significant improvement in PROs from preoperative scores at 1 year postoperatively, which is maintained at the 5-year longterm follow-up time point. Although patients have maintained improvements in mHHS and NAHS scores at 5-year follow up, the decrease in MCID achievement rate from 1 - to 5-year outcomes suggests there is a significant, clinically relevant decrease in clinical outcomes. In addition, after excluding known detrimental factors such as labral debridement and microfracture, the current results demonstrate the negative impact of increasing BMI and increasing age alongside the positive impact of cam resection on clinical outcomes, as demonstrated in the linear and binary logistic regression analyses. However, as the survival curves demonstrate, there is an early prevalence of requiring secondary arthroscopic or open soft tissue procedures after index hip arthroscopy at mid-term follow-up.

Previous long-term studies have demonstrated comparable positive outcomes after hip arthroscopy for FAI. ${ }^{2,6-12,23}$ Three studies by Domb et al. $(94,60$, and 42 patients, respectively) report improvements at both 2- and 5-year outcomes with no significant deterioration from 2 to 5 years in regard to PROs. ${ }^{6,10,11}$ While this current study was also unable to find statistical decreases from short-term (1-year) to long-term (5year) PROs in regard to cohort means of mHHS and NAHS in the analysis, the MCID analysis demonstrated that fewer patients achieved a sustained meaningful improvement from baseline preoperative scores at the time of 5-year follow-up as compared with both the 1year time point, which is arguably more clinically relevant than comparing mean absolute scores of the entire cohort. ${ }^{16}$ Despite significantly declining MCID rates in the current study, absolute rates remained superior at last follow-up as compared with Perets et al.; however, their cohort assessed patients older than the age of 50 years, many of whom underwent labral debridement, as compared with this current all-age inclusive cohort. ${ }^{11}$
A

Patient Acceptable Symptomatic State Rates

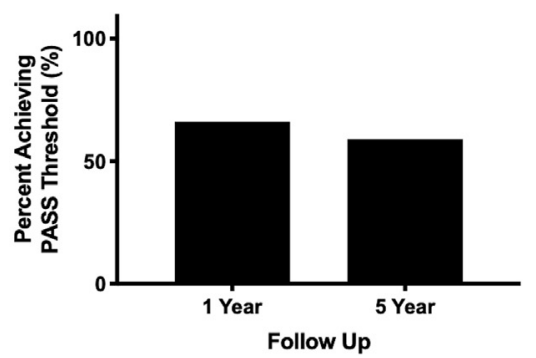

B

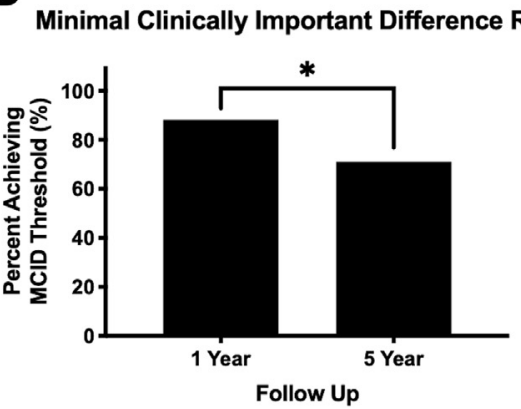

Fig 4. (A) PASS rates at 1-year and 5-year follow-up time points after index hip arthroscopy. (B) MCID rates at 1 -year and 5-year follow-up time points. MCID rate at the 5-year time point was significantly lower than 1 year. ${ }^{*} P$ $<$.01. (MCID, minimal clinically important difference; PASS, patient acceptable symptomatic state.) 
Although Domb et al. report a similar rate of secondary arthroscopic procedures and conversion to THA in their series assessing arthroscopic labral base repair, they quote a much greater conversion rate to THA in their series assessing arthroscopic acetabular microfracture and in patients older than age 50 years as compared with the current study. ${ }^{6,10,11}$ However, this studied cohort did not contain any patients receiving microfracture as compared with their series alongside the difficulty in comparing cohorts with a mean age of 41 (current study) versus 55.2. ${ }^{10,11}$ Additional areas for discrepancy between the studies of Domb et al. and this study is the nature of statistical analysis as the current study contains more comprehensive repeated-measures ANOVA analysis rather than isolated pairwise analyses of PROs at various time points as in the studies of Domb et al. Further, the current study uses Kaplan-Meier survival analysis accounting for censorship and loss to follow-up rather than absolute rates of conversion to secondary procedure and THA at follow-up time points, which provide a more accurate report of procedure survivability. Lastly, as opposed to the aforementioned studies, the current study excluded any patient with bilateral hip arthroscopy in this series, as this is a major confounder of PROs, given patients may experience symptoms from the contralateral hip when reporting outcomes for the hip in question during follow-up.

To date, Menge et al. ${ }^{2}$ have successfully reported large cohort $(\mathrm{n}=145)$ 10-year follow-up outcomes regarding hip arthroscopy for FAI treatment. Similar to Domb et al. they report a greater rate of THA conversion in subjects requiring acetabular microfracture, older age, and hip joint space narrowing indicative of osteoarthritis; however, PROs were improved from preoperative scores at 10-year assessment. ${ }^{2}$ Notably, the current study demonstrate much lower THA conversion rate at 5 -year outcomes $(10 \%)$ as compared with Menge et al. $(>20 \%$ ), but this cohort also does not include individuals with joint space less than $2 \mathrm{~mm}$, labral debridement, or microfracture which likely could account for the discrepancy. ${ }^{2}$

In regard to absolute rates of revision secondary procedures and conversion to THA, aside from the aforementioned studies, there is a significant range quoted in the existing literature. Skendzel et al. ${ }^{12}$ quote as high as $86 \%$ of patients with joint space narrower than $2 \mathrm{~mm}$ underwent conversion to THA at 5-year follow-up whereas only $16 \%$ converted with joint space $>2 \mathrm{~mm}$. While they demonstrate a lower mean postoperative PRO score in patients with less versus preserved joint space as well, mean PRO scores in both sub-cohorts improved significantly from baseline. ${ }^{12}$ Conversely, Chen et al. ${ }^{9}$ report $14 \%$ revision procedure and $1.9 \%$ conversion to THA rate within 5 years after hip arthroscopy for FAI in a runner cohort. However, mean age (32.4 years), BMI (22.9), and inclusion criteria of mid- to long-distancing running in their cohort as opposed to this study mean age (41 years), BMI (25.1), and overall patient characteristics differ substantially. ${ }^{9}$ Interestingly, Chen et al. ${ }^{8}$ also report in another study assessing 5-year outcomes after selective debridement versus labrum repair with the intent to preserve the labrum that the group undergoing debridement $(\mathrm{n}=101)$ only had a $4 \%$ conversion to THA while still having a $13.9 \%$ secondary procedure rate (with the caveat of these rates being percentage rates rather than Kaplan-Meier based survival rates). Lastly, Domb et al. ${ }^{7}$ also report $31.6 \%$ versus $11.6 \%$ conversion to THA at 5 -years based on increased Tönnis grade as a subanalysis of their patient series.

Unfortunately, while studies assessing long-term ( $\geq 5$ year) outcomes for hip arthroscopy treating FAI all demonstrate improvements in PROs, ${ }^{6,7,9-12,23}$ cohort heterogeneity based on inclusion criteria and chosen PRO metrics are major limitations to comparing studies with one another. Further, studies all demonstrate overall improvement in symptomatology while incidence of revision and conversion to THA increase with time from index procedure. This current analysis demonstrates that MCID rates actually deteriorate after initial postoperative improvement despite sustained improvement in PROs.

As discussed, hip joint space signifying progression of arthritis, labral debridement versus repair, presence of microfracture, dysplastic hip changes on radiographs, and increasing age have all been associated with worse long-term outcomes after hip arthroscopy. 2,6,7,9-12 To date, increasing BMI has only been associated with poorer long-term outcomes in a cohort older than the age of 50 years. ${ }^{11}$ After excluding the aforementioned known factors negatively affecting outcomes, the current study provides evidence demonstrating a correlation with increasing BMI and declining 5-year MCID and NAHS outcomes in the general FAI population. Further, the logistic and linear regressions support cam resection and younger age as positive prognostic variables in relation to PROs.

The strengths of this paper include the consecutive nature of cases, generalizable cohort without major excluding factors, the exclusion of any bilateral hip cases (which is a limitation of the former studies in the literature) to properly isolate outcomes after surgery, the statistical analysis comparing PROs in a repeated fashion along two major timepoints of follow up, and use of multiple clinically relevant PRO metrics. Further, our exclusion of labral debridement and microfracture cases increase the homogeneity of our cohort allowing a more representative depiction of outcomes after hip arthroscopy. Lastly, the linear regression assessing 5year PROs in tandem with the logistic regression assessing 5-year MCID achievement rate highlight the importance of BMI as a prognostic factor. 


\section{Limitations}

As outlined in our results, the comparison between 1 -year and 5-year mHHS and NAHS values in our population was underpowered given 343 and 984 patients are needed to allow for acceptable type II error rates in our analysis. However, with effect sizes of 2.9 and 1.8 in mHHS and NAHS, respectively, the clinical relevance of these differences would be negligible. Our eligible consecutive case inclusion rate was not perfect adding a nonresponse bias to outcomes and there is always a level of response bias in any study using PRO metrics. Further, there was a limited number of cases with all time points available for our serial comparisons, resulting in a minor number of cases being excluded from a subset of the statistical analyses performed in this study. Additional limitations stem from the single-surgeon nature of this study as well as. The fact that our population is significantly older than that of other cohorts published on the literature likely may have a role with the increased rate of revision procedures in the current study. 5,9,11,14

\section{Conclusions}

There is a decline in MCID at 5-year follow up after hip arthroscopy for FAI. Lower BMI, younger age, and cam resection are associated with positive outcomes. There is excellent index procedure survivability and excellent THA prevention rate.

\section{References}

1. Nho SJ, Beck EC, Nwachukwu BU, et al. Survivorship and outcome of hip arthroscopy for femoroacetabular impingement syndrome performed with modern surgical techniques. Am J Sports Med 2019;47:1662-1669.

2. Menge TJ, Briggs KK, Dornan GJ, McNamara SC, Philippon MJ. Survivorship and outcomes 10 years following hip arthroscopy for femoroacetabular impingement: Labral debridement compared with labral repair. J Bone Joint Surg Am 2017;99:997-1004.

3. Sogbein OA, Shah A, Kay J, et al. Predictors of outcomes after hip arthroscopic surgery for femoroacetabular impingement: A systematic review. Orthop J Sports Med 2019;7:2325967119848982.

4. Nwachukwu BU, Chang B, Fields K, et al. Outcomes for arthroscopic treatment of anterior inferior iliac spine (subspine) hip impingement. Orthop J Sports Med 2017;5: 2325967117723109.

5. Philippon MJ, Schroder ESBG, Briggs KK. Hip arthroscopy for femoroacetabular impingement in patients aged 50 years or older. Arthroscopy 2012;28:59-65.

6. Domb BG, Yuen LC, Ortiz-Declet V, Litrenta J, Perets I, Chen AW. Arthroscopic labral base repair in the hip: 5year minimum clinical outcomes. Am J Sports Med 2017;45:2882-2890.

7. Domb BG, Chaharbakhshi EO, Rybalko D, Close MR, Litrenta J, Perets I. Outcomes of hip arthroscopic surgery in patients with tonnis grade 1 osteoarthritis at a minimum 5-year follow-up: a matched-pair comparison with a Tonnis grade 0 control group. Am J Sports Med 2017;45: 2294-2302.

8. Chen AW, Yuen LC, Ortiz-Declet V, Litrenta J, Maldonado DR, Domb BG. Selective debridement with labral preservation using narrow indications in the hip: Minimum 5-year outcomes with a matched-pair labral repair control group. Am J Sports Med 2018;46:297-304.

9. Chen AW, Craig MJ, Yuen LC, Ortiz-Declet V, Maldonado DR, Domb BG. Five-year outcomes and return to sport of runners undergoing hip arthroscopy for labral tears with or without femoroacetabular impingement. Am J Sports Med 2019;47:1459-1466.

10. Domb BG, Rybalko D, Mu B, Litrenta J, Chen AW, Perets I. Acetabular microfracture in hip arthroscopy: Clinical outcomes with minimum 5-year follow-up. Hip Int 2018;28:649-656.

11. Perets I, Chaharbakhshi EO, Mu B, et al. Hip arthroscopy in patients ages 50 years or older: minimum 5-year outcomes, survivorship, and risk factors for conversion to total hip replacement. Arthroscopy 2018;34:3001-3009.

12. Skendzel JG, Philippon MJ, Briggs KK, Goljan P. The effect of joint space on midterm outcomes after arthroscopic hip surgery for femoroacetabular impingement. Am J Sports Med 2014;42:1127-1133.

13. Beck EC, Drager J, Nwachukwu BU, et al. Patients with borderline hip dysplasia achieve clinically significant improvement after arthroscopic femoroacetabular impingement surgery: A case-control study with a minimum 5-year follow-up. Am J Sports Med 2020;48: 1616-1624.

14. Maldonado DR, Chen JW, Kyin C, et al. Hips with acetabular retroversion can be safely treated with advanced arthroscopic techniques without anteverting periacetabular osteotomy: Midterm outcomes with propensity-matched control group. Am J Sports Med 2020;48:1636-1646.

15. Litrenta JM, Mu BH, Chen AW, Perets I, Ortiz-Declet V, Domb BG. Arthroscopic labral treatment in adolescents: Clinical outcomes with minimum 5-year follow-up. Am J Sports Med 2019;47:870-875.

16. Cvetanovich GL, Weber AE, Kuhns BD, et al. Hip arthroscopic surgery for femoroacetabular impingement with capsular management: Factors associated with achieving clinically significant outcomes. Am J Sports Med 2018;46:288-296.

17. Kunze KN, Beck EC, Nwachukwu BU, Ahn J, Nho SJ. Early hip arthroscopy for femoroacetabular impingement syndrome provides superior outcomes when compared with delaying surgical treatment beyond 6 months. Am J Sports Med 2019:2038-2044.

18. Basques BA, Waterman BR, Ukwuani G, et al. Preoperative symptom duration is associated with outcomes after hip arthroscopy. Am J Sports Med 2019;47:131-137.

19. Christensen CP, Althausen PL, Mittleman MA, Lee JA, McCarthy JC. The nonarthritic hip score: Reliable and validated. Clin Orthop Relat Res 2003:75-83.

20. Byrd JW, Jones KS. Prospective analysis of hip arthroscopy with 2-year follow-up. Arthroscopy 2000;16:578-587.

21. Chahal J, Van Thiel GS, Mather RC 3rd, et al. The patient acceptable symptomatic state for the modified Harris Hip score and hip outcome score among patients undergoing 
surgical treatment for femoroacetabular impingement. Am J Sports Med 2015;43:1844-1849.

22. Stone AV, Beck EC, Malloy $\mathrm{P}$, et al. Preoperative predictors of achieving clinically significant athletic functional status after hip arthroscopy for femoroacetabular impingement at minimum 2-year follow-up. Arthroscopy 2019;35:3049-3056 e3041.

23. Byrd JW, Jones KS. Prospective analysis of hip arthroscopy with 10-year followup. Clin Orthop Relat Res 2010;468:741-746. 